\title{
Segmentation Techniques for Medical Images - An Appraisal
}

\author{
S. Rakoth Kandan \\ Research Scholar (Ph.D) \\ Computer Science and \\ Engineering, \\ Annamalai University \\ India
}

\author{
J. Sasikala \\ Assistant Professor \\ Computer Science and Engineering, \\ Annamalai University \\ India
}

\begin{abstract}
This Paper provides the various analyses of Image Segmentation techniques for any field of image processing based applications. Segmentation is considered as a basic need in image processing for find the lines, curves, boundaries, etc in an image. In order to classify the segmentation techniques such as GA, Neural Network, Soft Computing and various image segmentation techniques and their performances analysis is done. Based on the performance analysis of segmentation techniques has been analyzed and conclude that each technique as best under the various field.
\end{abstract}

\section{Keywords}

Image Segmentation, Neural network, GA, Soft Computing

\section{INTRODUCTION}

The image is a visual representation of things. Image Segmentation is an essential part of image processing. The first and important processing of segmentation are to divide the image into multiple sub-regions depends on the particular feature. Segmentation is used to find the lines, curves, boundaries in an image. Now a day's segmentation process is applying for medical field applications for treatment diagnosis and analyzing. Much work has been already done in image segmentation and algorithms for research work are carrying to overcome the segmentation problems to be efficient work.

In the process of image segmentation, medical images the required details depend on the clinical application of particular problem [1]. The basic purpose of image segmentation is to improve the visualization process for disease identification in clinical purpose. The basic Principle of image segmentation is similarity and discontinuity. Similarity properties objects are utilized to find out the regions of interest. Under discontinuity properties, detecting the boundaries among sub-regions through the use of similar properties. In medical image segmentation, facing many problems like noise, uniform intensity, and lack of diverse features.

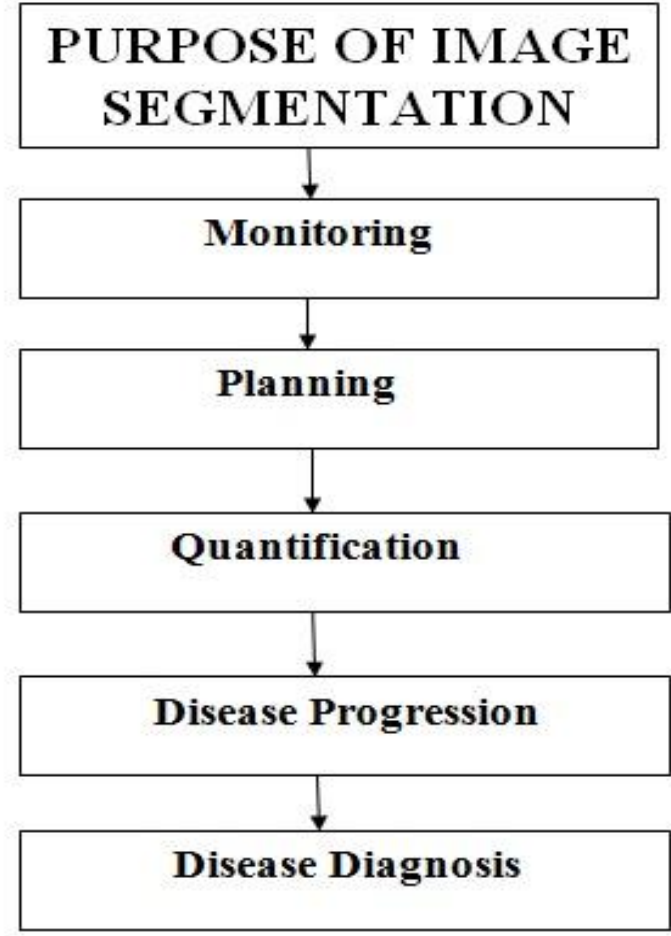

Fig 1: Purpose of Segmentation

If noise occurs in image uncertainty will occur which will not easy for image classification [2]. The value of intensity pixels will be reform because of noise in the image. Noise can occur in the image due to blurring, etc. To handle the uncertainty image segmentation plays a vital role.

\section{EDGE DETECTION TECHNIQUES}

Edge detection is one of the types of image segmentation. This is most common approaches way of finding the discontinuity it can be identified by the pixel and it will compare with the neighbor pixel. If suspect that two connected pixels have the same level of intensity distribution it will form the edge and it will not enough so they will form a closed path [3]. The basic principles of edge detection techniques are Filtering, Enhancement, and Detection. 


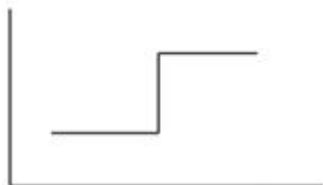

(a)

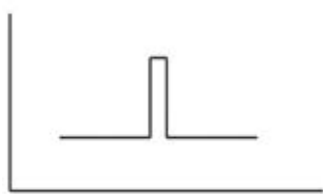

(c)

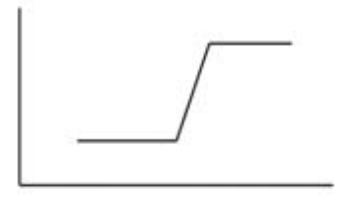

(b)

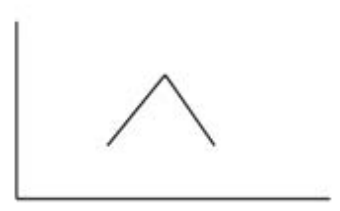

(d)
Figure 1: Type of Edges (a) Step Edge (b) Ramp Edge (c) Line Edge (d) Roof Edge

\subsection{Robert Edge Detection}

This operator is very simple and quick to compute, 2-D spatial gradient measurement on an image. The input to this operator is a grayscale image, the output of this operator performance complete magnitude of the image.

\begin{tabular}{|c|c|}
\hline 1 & 0 \\
\hline 0 & -1 \\
\hline
\end{tabular}

$\mathbf{G x}$

\begin{tabular}{|c|c|}
\hline 0 & 1 \\
\hline-1 & 0 \\
\hline
\end{tabular}

Gy

The calculation of the gradient magnitude is as follows

$$
|\mathbf{G}|=|\mathbf{G x}|+|\mathbf{G} \mathbf{y}|
$$

\subsection{Prewitt Edge Detection}

This operator is treated as a relevant way to calculate the magnitude and orientation of an image. Magnitude will be in $\mathrm{X}$ and $\mathrm{Y}$ directions this operator consists pair of $3 \times 3$ convolution kernel and it is limited to 8 possible orientations.

\begin{tabular}{|c|c|c|}
\hline-1 & 0 & +1 \\
\hline-1 & 0 & +1 \\
\hline-1 & 0 & +1 \\
\hline
\end{tabular}

$|\mathbf{G}|=\max (|\mathbf{G i}|: \mathbf{i}=1$ to $n)$

Here,

$\mathrm{G}_{\mathrm{i}}$-The response of the kernel $\mathrm{i}$ at the appropriate pixel position

$\mathrm{n}$-The number of convolution kernels.

\subsection{Sobel Edge Detection}

This operator locates the edges containing highest gradient. It consists pair of $3 \times 3$ convolution. Single kernel easily other rotated by $90^{\circ}$.

\begin{tabular}{|}
\begin{tabular}{|c|c|c|}
\hline-1 & 0 & +1 \\
\hline-2 & 0 & +2 \\
\hline-1 & 0 & +1 \\
\hline+1 & +2 & +1 \\
\hline 0 & 0 & 0 \\
\hline-1 & -2 & -1 \\
\hline
\end{tabular}
\end{tabular}

Approximate magnitude is computed by

$|\mathbf{G}|=|\mathbf{G x}|+|\mathbf{G y}|$

Sobel Edge Detection Method - Steps

Input: A Sample Image

Output: Detected Edges.

Step 1: Obtain the input image.

Step 2: The mask Gx, Gy is applied to the input image.

Step 3: The Sobel edge detection algorithm is applied.

Step 4: Gradient magnitude is calculated.

Step 5: Approximate magnitude is computed to obtain the result.

Step 6: The angle of the gradient vector is the output edges.

Table 1. Comparison Image Segmentation Operators

\begin{tabular}{|c|c|c|c|c|}
\hline Operators & Comparative Study & Parameter & Advantages & Disadvantages \\
\hline 1. Sobel & $\begin{array}{l}\text { Itproduces thin edges. } \\
\text { It does not offer } \\
\text { detailed information. }\end{array}$ & Threshold & $\begin{array}{l}\text { It is simple. Better } \\
\text { approximation to } \\
\text { gradient magnitude. }\end{array}$ & $\begin{array}{l}\text { Sensitivity to Noise. } \\
\text { Not accurate in } \\
\text { locating edges. } \\
\text { Accuracy descends } \\
\text { when magnitude of the } \\
\text { edges decreases. }\end{array}$ \\
\hline 2.Prewitt & $\begin{array}{c}\text { More sensitive to } \\
\text { honzontal and vertical } \\
\text { edgges. }\end{array}$ & Threshold & $\begin{array}{l}\text { Detection of edges and } \\
\text { their onientations are } \\
\text { high. }\end{array}$ & $\begin{array}{l}\text { Inaccurate. Size of the } \\
\text { coefficient and kemel } \\
\text { filter is fixed and } \\
\text { cannot be changedto a } \\
\text { given image. }\end{array}$ \\
\hline 3. Roberts & $\begin{array}{l}\text { Works with binary } \\
\text { images only. Does not } \\
\text { detect edges when a } \\
\text { minimal change in } \\
\text { gray scale value. }\end{array}$ & Threshold & $\begin{array}{l}\text { It is simple and fast. } \\
\text { Detects thicker edges. }\end{array}$ & $\begin{array}{l}\text { Localization is not } \\
\text { good.Weak response } \\
\text { to genuine edge. }\end{array}$ \\
\hline
\end{tabular}

\section{MODALITIES OF MEDICAL IMAGE} SEGMENTATION

The segmentation process is applied to many medical filed for disease identification. MRI brain images in this images high SNR it requires segmentation to find out the ROI. The main motives for segmenting these images are extracting the brain volume [4]. Medical applications differ based on disease, the Computed tomography images main motive for analyzing this images for liver problems, heart, abdominal, stomach. Contrast is not so good compare with previous image [5]. 
Ultrasound images are normally taken with a high rate of imperfection radiation is passed through the tissue so manual segmentation is done for this type of images [6]. Detection of the tumor is another important issue for physicians some algorithms are used for this type of images for detecting the tumor [7].

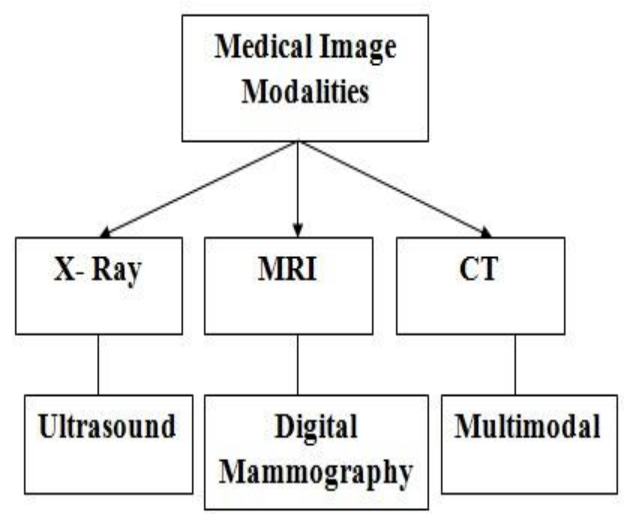

Figure 3: Types of Medical Image Modalities

\section{THRESHOLDING SEGMENTATION}

It is one of the simple methods for segmentation. Creating the binary images using grayscale images. It is further divided into three categories Local, Global, Merge. Local techniques fully depend on the pixels and neighborhoods. Global techniques are using the image histogram along with global texture properties [8]. Color images can segment using this method separate threshold should be set for all RGB components and need to combine all. Mostly HSV and HSL color models are using.

\subsection{Region Growing}

In this process, an ROI selection of initial seed points is the first work and adding the neighbor pixels to that particular region but it depends on the membership such as color. At first seed regions are identified based on a calculation of RGB components. First, seed point should have the minimum RGB component value four connected neighbors are used to grow the seed point [9]. The main application is to detect the tumor disease diagnosis it cannot utilize on its own some additional operations are required for region growing. A new method for detection of brain abnormalities is proposed on [10]. This technique can be applied based on the mean and Max-Min strategies [11]. The image should be dividing into uniform blocks first, then region growing should test for each block and add with adjacent blocks.

\subsection{Region Splitting and Merging}

Split and merge technique is the opposite of the region growing. This technique works on the whole image. Region splitting is a top-down approach. It begins with a whole image and divides it up such that the segregated parts are more homogenous than the whole. Splitting alone is insufficient for reasonable segmentation as it severely limits the shapes of segments. Hence, a merging phase after the splitting is always desirable, which is termed as the split-and-merge algorithm. Any region can be split into sub-regions, and the appropriate regions can be merged into a region. Rather than choosing seed points, the user can divide an image into a set of arbitrary unconnected regions and then merge the regions in an attempt to satisfy the conditions of reasonable image segmentation
[12]. Region splitting and merging is usually implemented with a theory based on quadtree data.

\subsection{Region splitting Method}

1. Let $\mathrm{R}$ represent the entire image. Select a predicate $\mathrm{P}$.

2. Split or subdivide the image successively into smaller and smaller quadrant regions.

The splitting technique has a convenient representation in the form of a structure called a quadtree. In a quadtree, the root of the tree corresponds to the entire image and each node corresponds to the subdivision.

\subsection{Region Merging Method}

Merge any adjacent regions that are similar enough. The procedure for split and merge is given.

1. Start with the whole image.

2. If the variance is too large, break it into quadrants.

3 . Merge any adjacent regions that are similar enough.

4. Repeat step (2) and (3) iteratively until no more splitting or merging occurs.

This technique requires the input data to be organized into a pyramidal grid structure of regions, with each region organized in groups of four in the case of $2 \mathrm{D}$, and of eight in the case of 3D.

\section{SOFT COMPUTING TECHNIQUES}

In medical image segmentation, automatic diagnosis of disease identification plays a vital role [13]. Soft computing techniques have many applications are use for segmentation. These are Fuzzy Based Approach, Genetic Algorithm, and Neural Networks.

\subsection{Fuzzy Based Approach}

There are many research possibilities is going on for an increase this type based edge detection techniques by defining the membership function representing the degree of edge in each neighbor [14]. These approaches consider as a true fuzzy approach if this concept used for additional change the membership values.

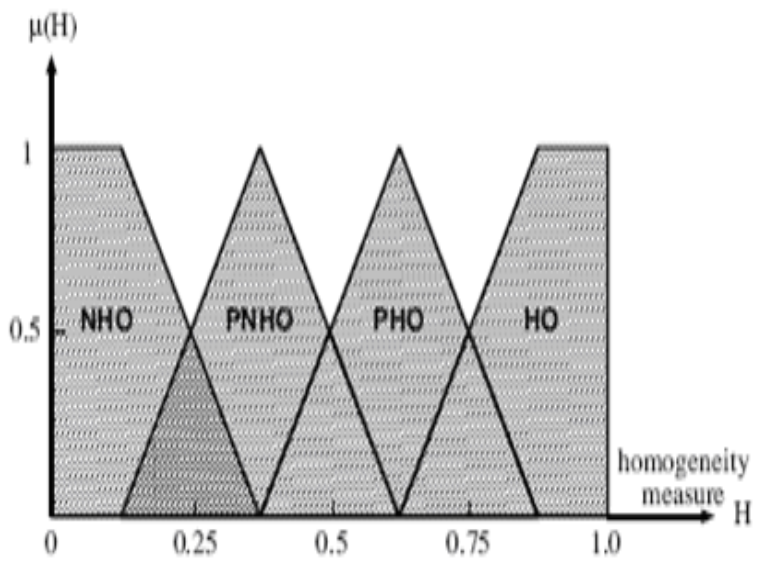

Figure 2: Fuzzy Sets for Homogeneity Inference

\subsection{Genetic Algorithm Approach}

The genetic algorithm includes three major processes: Selection, Crossover, and Mutation. The selection checks out each individual and keep only the fittest ones in the population [15]. According to small probability, some less fit could be select others are removed from the population. Crossover combines the two individuals to include new ones 
which will be better. Mutation operator causes for small changes in chromosome units for maintaining the population diversified during the optimization process. GAs are most extensive and details work for image segmentation the important reason for approach this technique is to deal with huge, complex search spaces somewhere only minimum amount of knowledge available about the objective function [16].

\subsection{A simple GA consists of five steps}

1. Start with a randomly generated population of $\mathrm{N}$ chromosomes, where $\mathrm{N}$ is the size of population, 1 - length of chromosome $\mathrm{x}$.

2. Calculate the fitness value of function $\varphi(x)$ of each chromosome $\mathrm{x}$ in the population.

3. Repeat until $\mathrm{N}$ offspring are created: a. a. probabilistically select a pair of chromosomes from the current population using the value of fitness function.

b. Produce an offspring $y_{i}$ using crossover and mutation operators, where $\mathrm{i}=1,2 \ldots \mathrm{N}$.

4. Replace current population with newly created one.

5. Go to step 2.

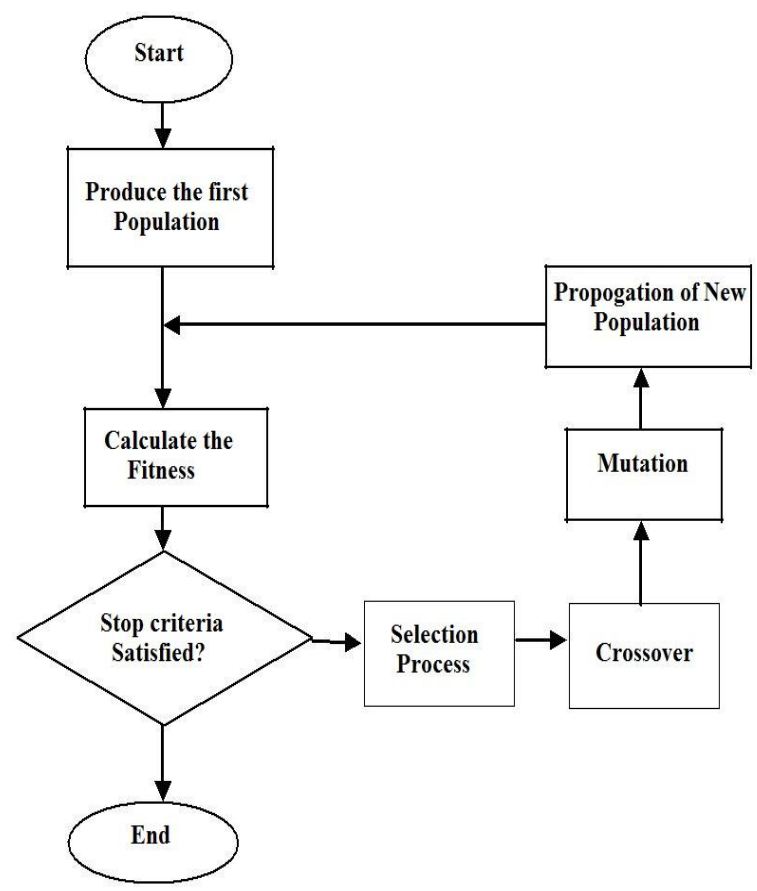

Figure 3: Flow Chart for Genetic Algorithm

\subsection{Neural Network Approach}

Neural Network is formed by several elements that are connected by various links with variable weights [17]. For pattern recognition techniques ANN is widely applied for medical image segmentation. Neural Network based on simulation of life, in particular, the human brain's learning process, constitutes a huge number of parallel nodes. All nodes can execute some basic computing technique. The neural network can also reduce the requirements of expert intervention during the image segmentation process. This problem is prevalent in many age segmentation methods. Firstly, the image segmentation problem is converted into energy minimization or classification issues and so no. Then the issues are solved based on the neural network in this method. The neural network was trained with training sample set in order to determine the connection and weights between the nodes. Then the new images were segmented with trained neural network. Neural network segmentation method includes two important steps: feature extraction and image segmentation based on neural network.

\section{CONCLUSION}

In this paper brief idea of various image segmentation methods applied for medical image processing has been reviewed including medical image segmentation. Soft computing techniques namely, fuzzy based approach, Genetic Algorithm based approach, and Neural Network based approaches are applied in real-time medical images for segmentation purpose. In future, this paper may be taken as small guidance to the person interest to work in medical image segmentation environment and computer aided diagnosis disease identification. This can be extended to the classification, feature extraction concepts for disease identification in a medical environment.

\section{ACKNOWLEDGMENTS}

The authors gratefully acknowledge the authorities of Annamalai University for the facilities offered to carry out this work.

\section{REFERENCES}

[1] Zuva T, Oludayo OO, Ojo SO, Ngwira SM. Image segmentation, available techniques, developments, and open issues. Canadian Journal on Image Processing and Computer Vision 2011; 2(3): 2009.

[2] Al-amri SS, Kalyankar NV, Khamitkar SD. A Comparative Study of Removal Noise from Remote Sensing Image, International Journal of Computer Science Issues (IJCSI) 2010; 7(1)

[3] Li BN, Chui CK, Chang S, Ong SH. Integrating spatial fuzzy clustering with level set methods for automated medical image segmentation, Computers in Biology and Medicine. 2011; 41(1): 1-10.

[4] Masood S, Sharif M, Yasmin M, Raza M, Mohsin S. Brain Image Compression: A Brief Survey, Research Journal of Applied Sciences. 2013; 5

[5] Xiao-juan C, Dan L, editors. Medical image segmentation based on threshold SVM. Biomedical Engineering and Computer Science (ICBECS), 2010 International Conference on; 2010: IEEE.

[6] Aja-Fernandez S, Vegas-Sanchez-Ferrero G, Fernandez M, editors. Soft thresholding for medical image segmentation, Engineering in Medicine and Biology Society (EMBC), 2010 Annual International Conference of the IEEE; 2010: IEEE.

[7] Shahzad A, Sharif M, Raza M, Hussain K. Enhanced watershed image processing segmentation, Journal of Information \& Communication Technology.2008; 2(1)01-9.

[8] Kenneth R. Castelman, "Digital image processing", Tsinghua Univ Press, 2003.

[9] Haider W, Sharif M, Raza M. Achieving accuracy in early stage tumor identification systems based on image segmentation and 3D structure analysis. Computer Engineering and Intelligent Systems 2011; 2(6): 96-102. 
[10] Siddique I, Bajwa IS, Naveed MS, Choudhary MA, editors. Automatic Functional Brain MR Image Segmentation using Region Growing and Seed Pixel, International Conference on Information \& Communications Technology, December;06.

[11] Bnar M. Ghafour, Nassir H. Salman. Medical Image Segmentation Based on Edge Detection Techniques. Vol. 3, Iss. 2, 2015.

[12] Upendra Kumar Chandra, Yogesh Bahendwar. Review on CAD-based System for Detection of Disease through Medical Image Processing. Vol. 4, Iss. 6, 2015.

[13] W. X. Kang, Q. Q. Yang, R. R. Liang, "The Comparative Research on Image Segmentation Algorithms", IEEE Conference on ETCS, pp. 703-707, 2009.

[14] N. Senthilkumaran and R. Rajesh, "A Study on Edge Detection Methods for Image Segmentation",
Proceedings of the International Conference on Mathematics and Computer Science (ICMCS-2009), 2009, Vol.I, pp.255-259.

[15] A. Borji, and M. Hamidi, "Evolving a Fuzzy Rule-Base for Image Segmentation", International Journal of Intelligent Systems and Technologies, 2007, pp.178-183.

[16] Xian Bin Wen, Hua Zhang and Ze Tao Jiang,"Multiscale Unsupervised Segmentation of SAR Imagery Using the Genetic Algorithm", Sensors, vol.8, 2008, pp.1704-1711.

[17] Hichem Talbi, Mohamed Batouche and Amer Draa,"A Quantum - Inspired Evolutionary Algorithm for Multiobjective Image Segmentation", International Journal of Mathematical, Physical and Engineering Sciences, Vol.1 No.2, 2007, pp.109-114. 